Article

\title{
及-Sitosterol Protects against Carbon Tetrachloride Hepatotoxicity but not Gentamicin Nephrotoxicity in Rats via the Induction of Mitochondrial Glutathione Redox Cycling
}

\author{
Hoi-Shan Wong, Ji-Hang Chen, Pou-Kuan Leong, Hoi-Yan Leung, Wing-Man Chan \\ and Kam-Ming Ko *
}

Division of Life Science, Hong Kong University of Science and Technology, Hong Kong, China; E-Mails: bchelenwong@ust.hk (H.-S.W.); cjh@ust.hk (J.-H.C.); eriol@ust.hk (P.-K.L.); hoiyan@ust.hk (H.-Y.L.); wingman@ust.hk (W.-M.C.)

* Author to whom correspondence should be addressed; E-Mail: bcrko@ust.hk; Tel.: +852-2358-7298.

External Editor: Maurizio Battino

Received: 14 September 2014; in revised form: 23 October 2014 / Accepted: 24 October 2014 / Published: 30 October 2014

\begin{abstract}
Previous findings have demonstrated that $\beta$-sitosterol (BSS), an active component of Cistanches Herba, protected against oxidant injury in H9c2 cardiomyocytes and in rat hearts by enhancing mitochondrial glutathione redox cycling, possibly through the intermediacy of mitochondrial reactive oxygen species production. We therefore hypothesized that BSS pretreatment can also confer tissue protection against oxidant injury in other vital organs such as liver and kidney of rats. In this study, the effects of BSS pretreatment on rat models of carbon tetrachloride $\left(\mathrm{CCl}_{4}\right)$ hepatotoxicity and gentamicin nephrotoxicity were investigated. The findings showed that BSS pretreatment protected against $\mathrm{CCl}_{4}$-induced hepatotoxicity, but not gentamicin nephrotoxicity in rats. The hepatoprotection afforded by BSS was associated with the improvement in mitochondrial glutathione redox status, presumably through the glutathione reductase-mediated enhancement in mitochondrial glutathione redox cycling. The hepatoprotection afforded by BSS was also accompanied by the improved mitochondrial functional ability in rat livers. The inability of BSS to protect against gentamicin nephrotoxicity was likely due to the relatively low bioavailability of BSS in rat kidneys. BSS may serve as potential mitohormetic agent for the prevention of oxidative stress-induced injury in livers.
\end{abstract}


Keywords: Cistanches Herba; $\beta$-sitosterol; carbon tetrachloride hepatotoxicity; gentamicin nephrotoxicity; glutathione redox cycling; mitochondria

\section{Introduction}

Kidney and liver are vital organs that participate actively in metabolic homeostasis, during which a number of reactive oxygen species (ROS) generating reactions are involved. The metabolic role of these organs makes them more vulnerable to oxidant injury [1-4]. In this connection, experimental findings showed that events such as ethanol consumption [5], drug exposure [6], acute exercise and aging $[7,8]$ were found to be associated with a shift of cellular redox environment to a more oxidized state in the kidney and liver, indicative of oxidative stress, with resultant impairment in cellular and mitochondrial functions [9]. Oxidative stress has been implicated in the pathogenesis of diseases such as chronic renal failure and hepatic fibrosis as well as the aging process [10-13]. As a consequence, interventions aimed at enhancing cellular antioxidant capacity, preferably by up-regulating cellular antioxidant defense mechanism [14], represent a rational approach for preventing the oxidative stress-induced tissue damage and retarding the aging process.

Cistanches Herba, the dried whole plant of Cistanche deserticola Y.C. Ma, is a "Yang-invigorating" tonic herb in Chinese medicine. Recently, a phytosterol, $\beta$-sitosterol (BSS, Figure S1, Table S1), was identified as an active component of Cistanches Herba in enhancing mitochondrial respiration and thereby improving glutathione redox status via an induction of mitochondrial ROS production in H9c2 cardiomyocytes. Our findings also demonstrated that BSS protected against oxidant injury in H9c2 cardiomyocytes $[15,16]$. Therefore, it was postulated that BSS increased mitochondrial electron transport and the associated sustained low level of mitochondrial ROS production in H9c2 cardiomyocytes. The increased mitochondrial ROS production was paralleled by an induction of mitochondrial uncoupling. The elevated level of mitochondrial ROS also caused an up-regulation of cellular glutathione redox cycling and the subsequent increased resistance to oxidant challenge [16]. The beneficial effects of BSS were further corroborated by their ability to protect against myocardial ischemia/reperfusion (I/R) injury ex vivo in rats, presumably via enhancing mitochondrial glutathione redox cycling [15-17]. Here, we endeavored to investigate whether BSS can also produce tissue protection against oxidant injury in rat livers and kidneys.

Carbon tetrachloride $\left(\mathrm{CCl}_{4}\right)$ hepatotoxicity and gentamicin nephrotoxicity are well established animal models to investigate potential hepatoprotective and nephroprotective agents [18-23]. In the present study, the effects of BSS pretreatment on $\mathrm{CCl}_{4}$ hepatotoxicity and gentamicin nephrotoxicity were investigated in rats. Mitochondrial glutathione redox status and mitochondrial functional ability in rat kidney and liver tissues were also examined. 


\section{Results}

\subsection{Effects of BSS on $\mathrm{CCl}_{4}$ Hepatotoxicity in Rats}

$\mathrm{CCl}_{4}$ administration caused liver injury in rats, as indicated by significant increases in plasma alanine aminotransferase (ALT) and aspartate aminotransferase (AST) activities (by 14 and 16-fold, respectively), when compared with the non- $\mathrm{CCl}_{4}$ control (Figure 1). BSS $(35 \mu \mathrm{g} / \mathrm{kg}$ ) pretreatments protected against $\mathrm{CCl}_{4}$ hepatotoxicity, as evidenced by significant suppressions in the $\mathrm{CCl}_{4}$-induced elevation in plasma ALT and AST activities by $48 \%$ and $41 \%$, respectively, when compared with the respective untreated $\mathrm{CCl}_{4}$ control (Figure 1). $\mathrm{CCl}_{4}$-induced liver damaged was associated with a significant impairment in the mitochondrial glutathione redox status (by 49\%), as assessed by the reduced glutathione (GSH)/oxidized glutathione (GSSG) ratio, in rat livers (Figure 2a). While BSS pretreatment produced no detectable effect on mitochondrial glutathione redox status in non-CCl4-intoxicated rat livers (Figure 2a), BSS, at daily doses of $35 \mu \mathrm{g} / \mathrm{kg}$, partially reversed the $\mathrm{CCl}_{4}$-induced impairment in mitochondrial glutathione redox status by $61 \%$, when compared with the untreated $\mathrm{CCl}_{4}$ control (Figure 2a).

Figure 1. Effects of BSS on plasma ALT and AST activities in control and $\mathrm{CCl}_{4}$-intoxicated female rats. Plasma (a) ALT and (b) AST activity were measured as described in Materials and methods. Data were expressed in percent control with respect to the non- $\mathrm{CCl}_{4}$ control (plasma ALT activity $=41.2 \pm 7.6 \mathrm{mU} / \mathrm{L}$; plasma AST activity $=73.3 \pm 11.8 \mathrm{mU} / \mathrm{L}$ ). Values given are means \pm SEM, with $n=7$.

(a)

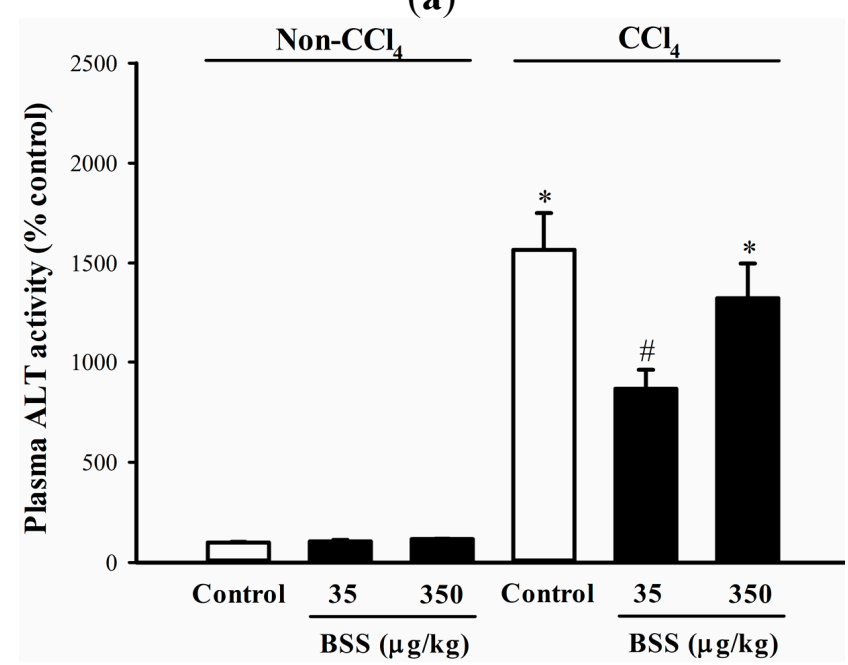

* Significantly different from the non- $\mathrm{CCl}_{4}$ control; ${ }^{*}$ significantly different from the $\mathrm{CCl}_{4}$ control $(p<0.05)$. (b)

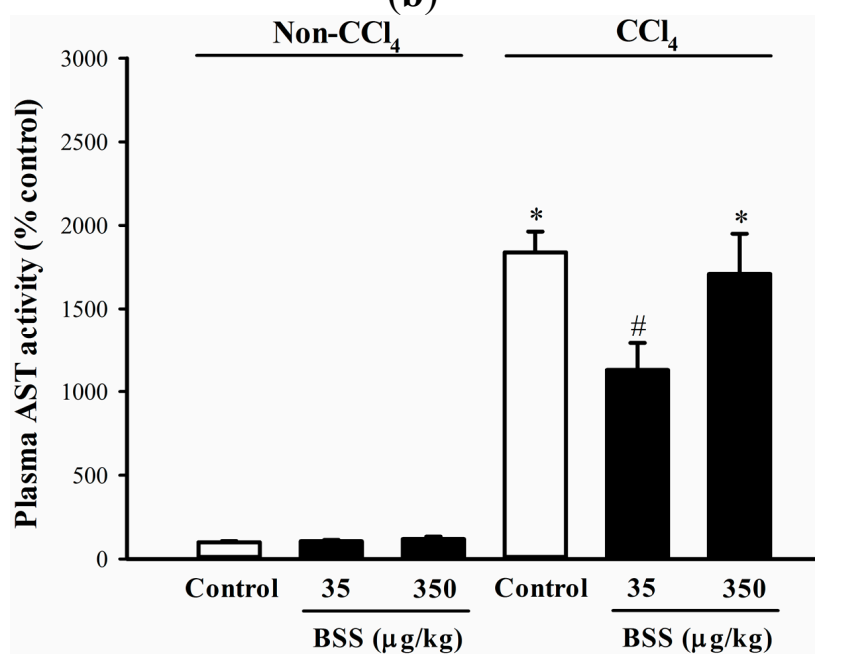

Both BSS and $\mathrm{CCl}_{4}$ challenge did not alter mitochondrial glutathione reductase (GR) and isocitrate dehydrogenase (ICDH) activities (Table 1). BSS $(35 \mu \mathrm{g} / \mathrm{kg})$ pretreatment increased the mitochondrial ICDH activity by $18 \%$ in $\mathrm{CCl}_{4}$-intoxicated rat livers, when compared with the unpretreated $\mathrm{CCl}_{4}$ control (Table 1). 
Figure 2. Effects of BSS on control and $\mathrm{CCl}_{4}$-intoxicated female rat livers. Data were expressed in percent control with respect to the non- $\mathrm{CCl}_{4}$ control ((a) mitochondrial $\mathrm{GSH} / \mathrm{GSSG}$ ratio $=9.6 \pm 0.3$; (b) tissue ATP level $=10.2 \pm 0.3 \mathrm{nmol} / \mathrm{mg}$ protein; $(\mathbf{c})$ ATP-GC value $=999.8 \pm 65.7 \mathrm{AU})$. Values given are means \pm SEM, with $\mathrm{n}=7$.

(a)

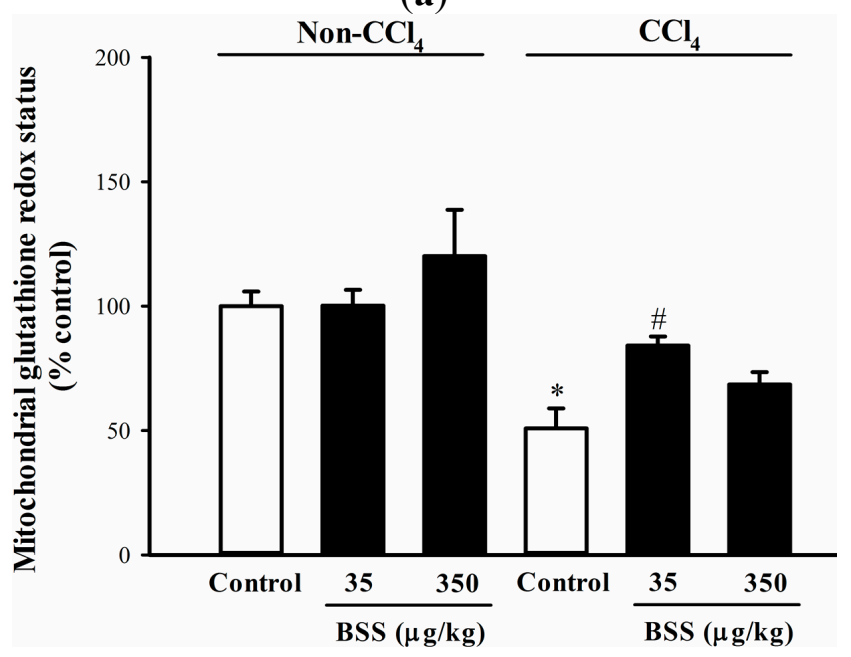

(b)

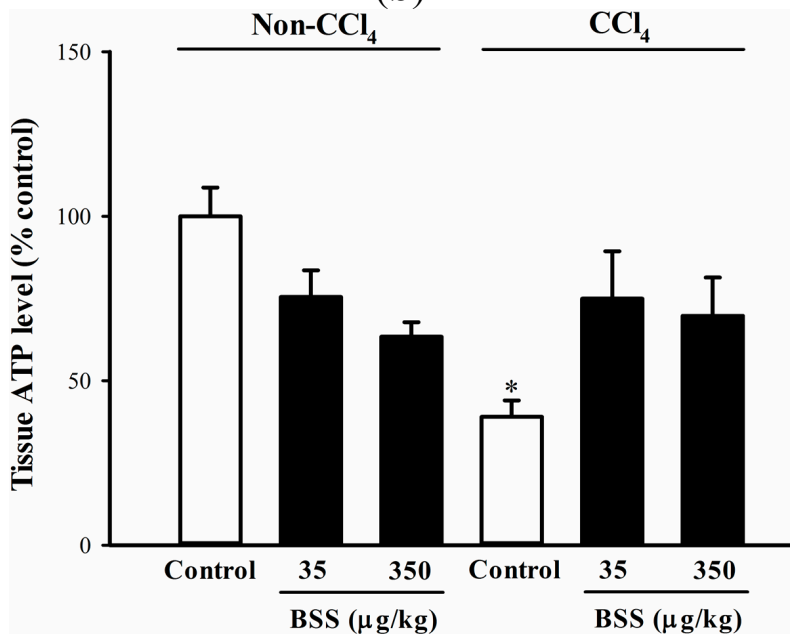

(c)

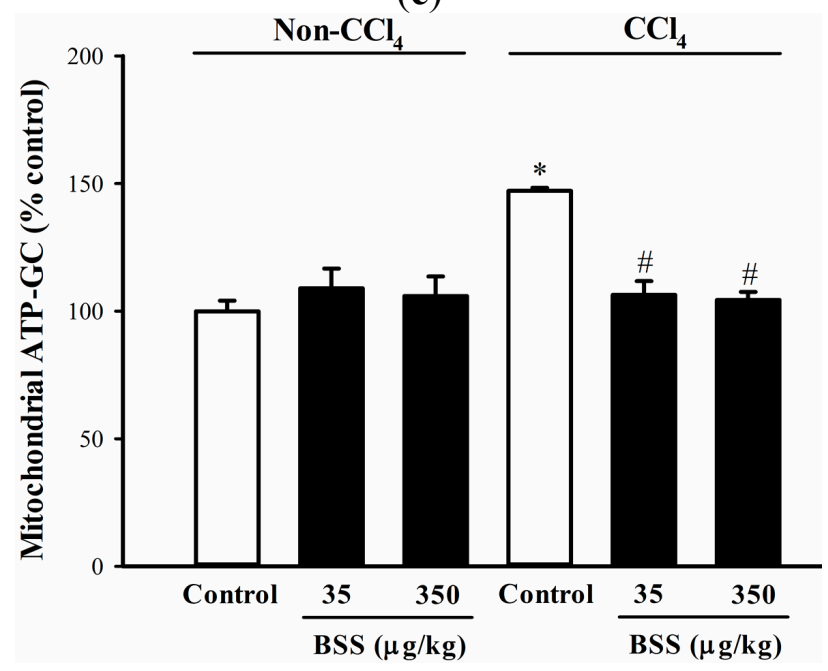

* Significantly different from the non- $\mathrm{CCl}_{4}$ control; ${ }^{\#}$ significantly different from the $\mathrm{CCl}_{4}$ control $(p<0.05)$.

Table 1. Effects of BSS pretreatment on mitochondrial GR and ICDH activity in livers of $\mathrm{CCl}_{4}$-intoxicated rats.

\begin{tabular}{|c|c|c|c|c|}
\hline \multirow[b]{2}{*}{$\%$ Non- $\mathrm{CCl}_{4}$ Control } & \multirow[b]{2}{*}{$\begin{array}{c}\text { Non-CCl} \\
\text { Control }\end{array}$} & \multicolumn{3}{|c|}{$\mathrm{CCl}_{4}$} \\
\hline & & Control & $\begin{array}{c}\text { BSS } \\
(35 \mu \mathrm{g} / \mathrm{kg})\end{array}$ & $\begin{array}{c}\text { BSS } \\
(350 \mu \mathrm{g} / \mathrm{kg})\end{array}$ \\
\hline Mitochondrial GR Activity & $100.0 \pm 2.9$ & $98.2 \pm 5.1$ & $92.9 \pm 9.5$ & $82.8 \pm 9.2$ \\
\hline Mitochondrial ICDH Activity & $100.0 \pm 2.2$ & $104.5 \pm 3.4$ & $117.5 \pm 6.0 *, \#$ & $102.1 \pm 3.7$ \\
\hline
\end{tabular}

Data were expressed in percent control with respect to the non- $\mathrm{CCl}_{4}$ control (mitochondrial GR activity $=7.1 \pm 0.3 \mathrm{mU} / \mathrm{mg}$ protein; mitochondrial ICDH activity $=49.8 \pm 1.2 \mathrm{mU} / \mathrm{mg}$ protein). Values given are means \pm SEM, with $\mathrm{n}=7$. ${ }^{*}$ Significantly different from the non- $\mathrm{CCl}_{4}$ control; ${ }^{\#}$ significantly different from the $\mathrm{CCl}_{4}$ control $(p<0.05)$. 
BSS $(350 \mu \mathrm{g} / \mathrm{kg})$ pretreatment significantly decreased the tissue ATP level by $37 \%$ in rat livers under non-challenged condition (Figure $2 \mathrm{~b}$ ). $\mathrm{CCl}_{4}$ intoxication caused significant reductions in tissue ATP level by $68 \%$ in rat livers (Figure $2 \mathrm{~b}$ ). BSS (35 and $350 \mu \mathrm{g} / \mathrm{kg}$ ) pretreatment partially reversed the $\mathrm{CCl}_{4}$-induced depletion of tissue ATP by 60 and 51\%, respectively, in rat livers (Figure $2 \mathrm{~b}$ and Table 2).

BSS treatment produced no detectable change in mitochondrial ATP generation capacity (ATP-GC) in livers of non- $\mathrm{CCl}_{4}$-intoxicated rats. Mitochondrial ATP-GC was increased after the $\mathrm{CCl}_{4}$ challenge by $55 \%$, when compared with the non- $\mathrm{CCl}_{4}$ control (Figure $2 \mathrm{c}$ ). The $\mathrm{CCl}_{4}$-induced elevation in mitochondrial ATP-GC was completely suppressed by BSS pretreatment (Figure 2c).

Table 2. Effects of BSS pretreatment on $\mathrm{CCl}_{4}$-induced hepatic ATP depletion in rats.

\begin{tabular}{cccc}
\hline & \multicolumn{3}{c}{$\mathbf{C C l}_{\mathbf{4}}$} \\
\cline { 2 - 4 }$\%$ & \multirow{2}{*}{ Control } & $\begin{array}{c}\text { BSS } \\
(\mathbf{3 5} \boldsymbol{\mu g} / \mathbf{k g})\end{array}$ & $\begin{array}{c}\text { BSS } \\
\mathbf{( 3 5 0} \boldsymbol{\mu g} / \mathbf{k g})\end{array}$ \\
\hline $\mathbf{C C l}_{4}$-induced Hepatic ATP Depletion & $68.0 \pm 4.9$ & $0.5^{\#} \pm 15.4$ & $-6.4^{\#} \pm 12.5$ \\
\hline
\end{tabular}

Data were expressed in percent control with respect to respective non- $\mathrm{CCl}_{4}$ animals. Values given are means $\pm \mathrm{SEM}$, with $\mathrm{n}=7$. " Significantly different from the $\mathrm{CCl}_{4}$ control $(p<0.05)$.

\subsection{Effects of BSS on Gentamicin Nephrotoxicity in Rats}

Gentamicin administration caused kidney injury in rats, as indicated by significant increases in blood urea nitrogen (BUN) and blood creatinine levels (by 3 and 2-fold, respectively), when compared with the non-gentamicin control (Figure 3). BSS produced no detectable effect on the gentamicin-induced elevations in BUN and blood creatinine level (Figure 3).

Figure 3. Effects of BSS on BUN and blood creatinine level in control and gentamicin-challenged rats. (a) BUN and (b) blood creatinine level were measured as described in Materials and methods. Data were expressed in percent control with respect to the non-gentamicin control (BUN level $=187.2 \pm 16.2 \mathrm{mg} / \mathrm{L}$; blood creatinine level $=4.5 \pm 0.3 \mathrm{mg} / \mathrm{L})$. Values given are means \pm SEM, with $\mathrm{n}=7$.

(a)
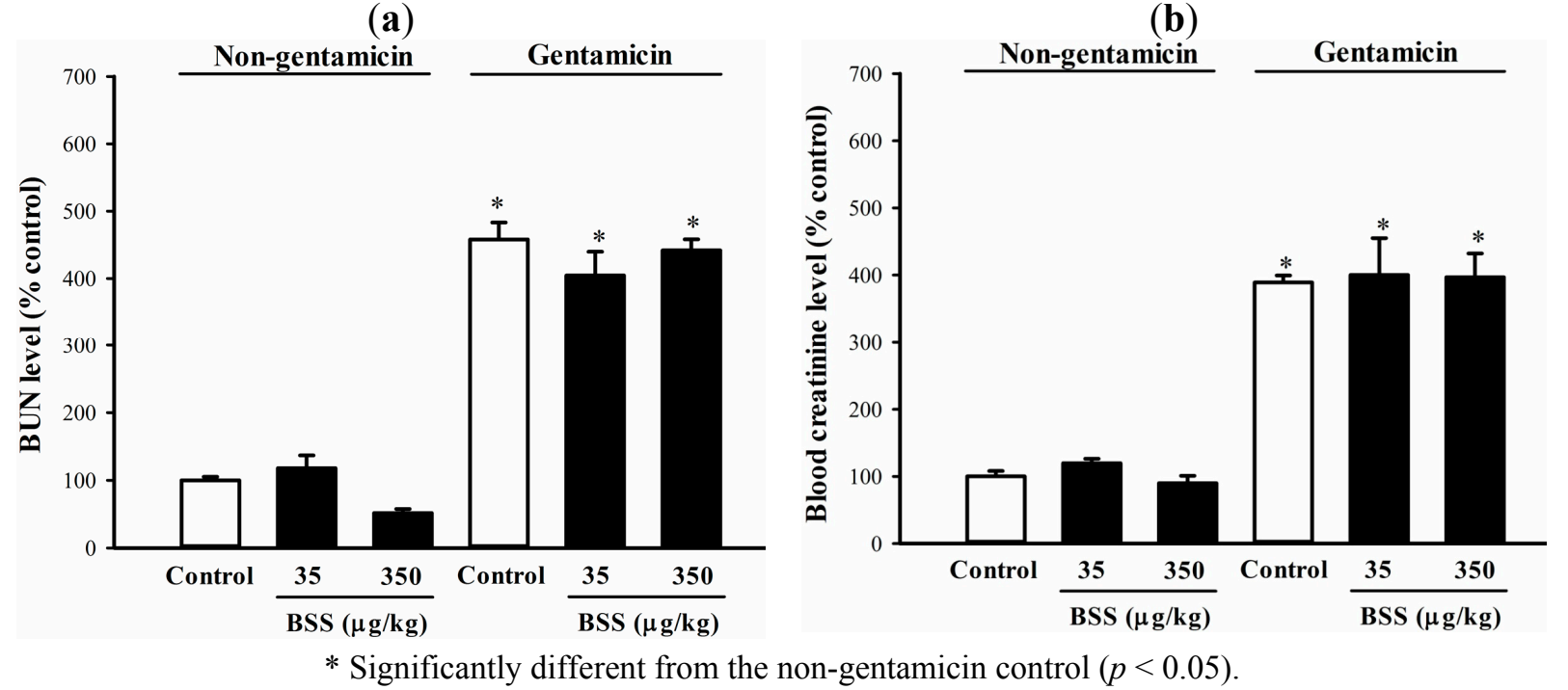
The gentamicin-induced kidney injury was found to be associated with a significant impairment in mitochondrial glutathione redox status, as indicated by a decrease (38\%) in the ratio of GSH to GSSG (Figure 4a). Consistent with the absence of nephroprotective effect, BSS pretreatment produced no detectable effect on mitochondrial glutathione redox status in rat kidneys under both non-challenged and challenged conditions, when compared with respective unpretreated controls (Figure 4a). Gentamicin challenge also significantly inhibited mitochondrial GR (40\%) and ICDH (45\%) activities in rat kidneys (Table 3). BSS pretreatment failed to produce any detectable effect on the gentamicin-induced suppression in mitochondrial GR and ICDH activity (Table 3).

Figure 4. Effects of BSS on control and gentamicin-challenged rat kidneys. (a) Mitochondrial glutathione redox status, (b) tissue ATP level and (c) mitochondrial ATP-GC were examined as described in Materials and methods. Data were expressed in percent control with respect to non-gentamicin control (mitochondrial GSH/GSSG ratio = $13.9 \pm 1.6$; tissue ATP level $=0.9 \pm 0.1 \mathrm{nmol} / \mathrm{mg}$ protein; ATP-GC value $=997.8 \pm 14.4 \mathrm{AU}$ ). Values given are means $\pm \mathrm{SEM}$, with $\mathrm{n}=7$.

(a)

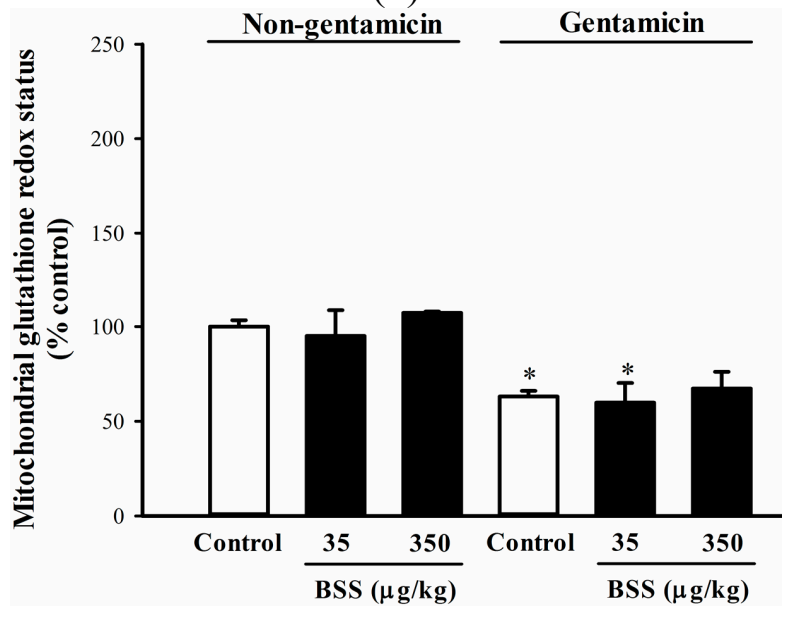

(b)

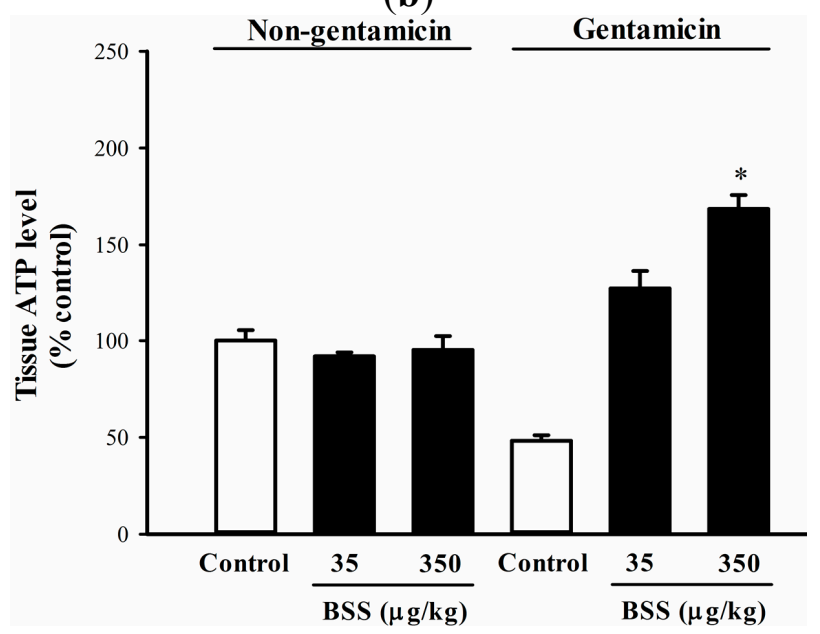

(c)

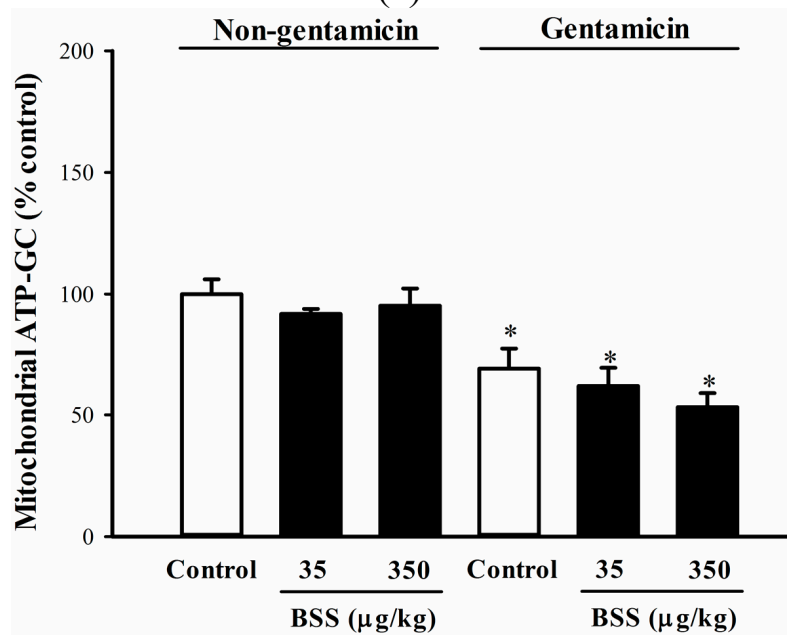

* Significantly different from the non-gentamicin control; * significantly different from the gentamicin control $(p<0.05)$. 
Table 3. Effects of BSS pretreatment on mitochondrial GR and ICDH activity in kidneys of gentamicin-challenged rats.

\begin{tabular}{lcccc}
\hline \multirow{2}{*}{$\%$ Non-gentamicin Control } & Non-gentamicin & \multicolumn{3}{c}{ Gentamicin } \\
\cline { 3 - 5 } & Control & Control & BSS & BSS \\
$\mathbf{( 3 5} \boldsymbol{\mu g} / \mathbf{k g})$ & $\mathbf{( 3 5 0} \boldsymbol{\mu g} / \mathbf{k g})$ \\
\hline Mitochondrial GR Activity & $100.0 \pm 4.0$ & $59.6 \pm 6.1 *$ & $63.2 \pm 3.9 *$ & $61.7 \pm 4.7 *$ \\
\hline Mitochondrial ICDH Activity & $100.0 \pm 3.6$ & $54.5 \pm 8.0 *$ & $61.0 \pm 6.5 *$ & $47.5 \pm 2.8 *$ \\
\hline Data were expressed in percent control with respect to the non-gentamicin control (mitochondrial GR \\
activity $=11.6 \pm 0.5 \mathrm{mU} / \mathrm{mg}$ protein; mitochondrial ICDH activity $=17.9 \pm 0.5 \mathrm{mU} / \mathrm{mg}$ protein). Values given are \\
means \pm SEM, with $\mathrm{n}=7$. * Significantly different from the non-gentamicin-challenged control $(p<0.05)$.
\end{tabular}

Gentamicin challenge caused a significant depletion of tissue ATP level (by 57\%) in rat kidneys, when compared with the respective non-gentamicin control (Figure 4b). While BSS pretreatments produced no detectable change in tissue ATP level in rat kidneys under non-challenged condition (Figure $4 \mathrm{~b}$ ), it (35 and $350 \mu \mathrm{g} / \mathrm{kg}$ ) caused increases in tissue ATP level (by $30 \%$ and $68 \%$, respectively) over the non-gentamicin control value in gentamicin-challenged rat kidneys (Figure 4b and Table 4). In regard to mitochondrial function, gentamicin challenge caused a significant decrease in mitochondrial ATP-GC by $35 \%$ in rat kidneys, when compared with the respective unpretreated non-gentamicin control (Figure 4c). BSS pretreatment did not produce detectable change in mitochondrial ATP-GC in rat kidneys under both non-challenged and challenged conditions, when compared with respective unpretreated controls (Figure 4c).

Table 4. Effects of BSS pretreatment on gentamicin-induced renal ATP depletion in rats.

\begin{tabular}{cccc}
\hline \multirow{2}{*}{$\%$} & \multicolumn{3}{c}{ Gentamicin } \\
\cline { 2 - 4 } & Control & BSS & BSS \\
& $(35 \mu \mathbf{g} / \mathbf{k g})$ & $\mathbf{( 3 5 0} \boldsymbol{\mu g} / \mathbf{k g})$ \\
\hline Gentamicin-induced Renal ATP Depletion & $57.1 \pm 2.6$ & $-35.6^{\#} \pm 8.4$ & $-73.4^{\#} \pm 7.1$ \\
\hline
\end{tabular}

Data were expressed in percent control with respect to respective non-gentamicin-challenged animals. Values given are means \pm SEM, with $\mathrm{n}=7$. ${ }^{\#}$ Significantly different from the gentamicin-challenged control $(p<0.05)$.

\section{Discussion}

BSS was previously identified as an active component of Cistanches Herba in enhancing mitochondrial respiration and thereby inducing mitochondrial glutathione redox cycling, presumably via the intermediacy of mitochondrial ROS production in H9c2 cardiomyocytes and rat hearts $[15,16]$ (Figure S1, Table S1). Previous findings have also demonstrated that long-term treatment with BSS produced protective effect against myocardial I/R injury in rats $[15,16]$. However, whether BSS pretreatment can protect against oxidant injury in other organs remains unclear. In the present study, we investigated the potential beneficial effects of BSS on oxidant injury in livers and kidneys of rats. The results showed that pretreatments with $\mathrm{BSS}$ protected against $\mathrm{CCl}_{4}$ hepatotoxicity but not gentamicin nephrotoxicity in rats. The hepatoprotection against $\mathrm{CCl}_{4}$ challenge in rat livers was associated with the enhancement in mitochondrial glutathione redox status. It has been demonstrated that a pro-oxidant shift in mitochondrial glutathione redox status (i.e., GSH/GSSG ratio) is associated 
with age-related cell death [24] and oxidative stress-induced tissue damages [25-28], presumably due to a disruption of redox signaling cascades. The maintenance of an optimal glutathione redox status is therefore critical for cell survival [29]. This postulation is consistent with our finding that the degree of tissue protection correlated with the extent of enhancement of mitochondrial glutathione redox status in BSS-pretreated $\mathrm{CCl}_{4}$-intoxicated rat livers. The hepatoprotective effect of BSS may be mediated by an up-regulation of mitochondrial glutathione redox cycling, which involves the regeneration of GSH from GSSG. In cellular environment, GSH is readily oxidized to GSSG for ROS detoxification, and it can be regenerated via a GR-catalyzed reaction at the expense of NADPH. In this regard, BSS pretreatment was found to increase mitochondrial $\mathrm{ICDH}$ activity in $\mathrm{CCl}_{4}$-intoxicated rat livers, wherein mitochondrial ICDH plays a pivotal role in supplying NADPH for the regeneration of GSH from GSSG and the subsequent improvement in mitochondrial glutathione redox status via the action of mitochondrial GR [30-32]. The degree of stimulation of ICDH activity was also found to correlate with an improvement in mitochondrial glutathione redox status in BSS-pretreated challenged animals, suggesting that BSS-induced up-regulation of mitochondrial glutathione redox cycling may be mediated by the activation of mitochondrial ICDH activity in rat livers. On the other hand, gentamicin-induced oxidant injury was found to be associated with the suppressions of mitochondrial GR and ICDH activities in rat kidneys. The absence of such association in $\mathrm{CCl}_{4}$-intoxicated rat livers may be explained by the differential susceptibility of kidney and liver to oxidant challenges [33,34], as was also observed in the present study. The incapability of BSS to ameliorate the gentamicin-induced impairment in mitochondrial glutathione redox status and suppression in mitochondrial GR and ICDH activities further supported the importance role of mitochondrial glutathione redox status in tissue protection against oxidant injury.

The observation that BSS induced hepatic ATP depletion under non-challenged condition indicated a sustained induction of mitochondrial uncoupling, whereas BSS failed to produce any effect on tissue ATP level in rat kidneys. The discrepant observation between livers and kidneys in regard to the effect of BSS on tissue ATP in non-challenged rats may be attributed at least in part to a relatively high resistance of kidney tissue to ATP depletion, as was the case in metabolic insults [35]. Both $\mathrm{CCl}_{4}$ and gentamicin challenge caused significant depletions in tissue ATP levels in rat livers and kidneys, presumably due to an impaired mitochondrial electron transport and oxidative phosphorylation. While BSS produced no detectable effect on mitochondrial ATP-GC in rat livers, the hepatoprotective effect of BSS was found to be associated with the reduction in the extent of $\mathrm{CCl}_{4}$-induced tissue ATP depletion (Table 2), which may be explained by a reduced extent of tissue/mitochondrial damage under oxidative stress conditions. The unexpected increases in renal ATP level in BSS-pretreated and gentamicin-challenged rats remain to be investigated (Figure $4 \mathrm{~b}$ and Table 4). It is possible, as reported by Devalaraja-Narashimha et al. [36] and Park et al. [37], that BSS may modulate renal energy homeostasis by its ability to degrade poly-(ADP-ribose) polymerase, an enzyme that induces a significant ATP depletion and cell death under oxidative stress condition.

Our previous findings on $\mathrm{H} 9 \mathrm{c} 2$ cardiomyocytes showed that the cytoprotective effect afforded by HCF1 (an active fraction of Cistanches Herba)/BSS against hypoxia/reoxygenation-induced apoptosis was mediated by the induction of mitochondrial glutathione redox cycling via the HCF1-induced mitochondrial ROS production secondary to increased mitochondrial electron transport $[15,16]$. Based on our current findings, we postulate that BSS pretreatment may up-regulate mitochondrial glutathione 
redox cycling by increasing mitochondrial ROS production, possibly through the stimulation of mitochondrial electron transport. Preliminary studies indicated that BSS may increase the fluidity of mitochondrial inner membrane and thereby stimulate mitochondrial electron transport (data not shown). The finding that $\mathrm{BSS}$ protected against $\mathrm{CCl}_{4}$ hepatotoxicity suggests its potential use as a mitohormetic agent to prevent oxidative stress-induced tissue injury in the liver, particularly during the aging process, in which increased prevalences of various liver diseases were observed [38-40]. While the BSS-containing HCF1 could protect against gentamicin nephrotoxicity in rats (unpublished data), the inability of BSS in ameliorating the gentamicin nephrotoxicity may be explained by its relatively low bioavailability, particularly when in the absence of other components in HCF1, in rat kidneys. It has been shown that BSS as well as other phytosterols, such as campesterol, exhibited a relatively low concentration and a short retention time in rat kidneys, when compared with rat livers [41]. While dietary compounds such as polyphenols and polyunsaturated fatty acids have been shown to improve mitochondrial function and regulate apoptosis process both in normal and pathological conditions [17], our recent and present findings of enhancement of mitochondrial ATP generation capacity and glutathione redox status by BSS demonstrate the potential beneficial effect of triterpenoids on mitochondrial energy generation and antioxidant mechanism [16].

\section{Experimental Section}

\subsection{Chemicals}

BSS (CAS 83-46-5) was purchased from Sigma (St. Louis, MO, USA). QuantiChrom ${ }^{\mathrm{TM}}$ Urea Assay kit (DIUR-500) and QuantiChrom ${ }^{\mathrm{TM}}$ Creatinine Assay kit (DICT-500) were purchased from BioAssay Systems (Hayward, CA, USA). ATPLite ${ }^{\mathrm{TM}}$ bioluminescence assay kit (\#6016941) was purchased from Perkin Elmer Inc. (Santa Clara, CA, USA). Assay kits for ALT activity and AST activity, and all other chemicals were purchased from Sigma.

\subsection{Animal Care}

Female Sprague-Dawley rats ( 8 weeks; 200 to $250 \mathrm{~g}$ ) were maintained under a 12-h dark/light cycle in an air/humidity controlled room at about $22{ }^{\circ} \mathrm{C}$, and allowed food and water ad libitum in the Animal and Plant Care Facilities (APCF) at HKUST. All experimental procedures were approved by the Research Practice Committee (HKUST) [42]. Rats were randomly divided into groups, with seven animals in each unless otherwise specified. In the experiment, rats were administered intragastrically with BSS, at daily doses of 35 and $350 \mu \mathrm{g} / \mathrm{kg}$, which were proven to be effective to protect against myocardial ischemia/reperfusion injury in rats [16], for 14 consecutive days. Control animals received vehicle (olive oil) only.

\section{3. $\mathrm{CCl}_{4}$ Hepatotoxicity}

$\mathrm{CCl}_{4}$ was intragastrically administered at a dose of $1 \mathrm{~mL} / \mathrm{kg}$ after the last dosing of $\mathrm{BSS}$, and vehicle was given to the non- $\mathrm{CCl}_{4}$-intoxicated rats. Twenty-four hours after $\mathrm{CCl}_{4}$ challenge, blood samples were drawn from phenobarbital-anesthetized animals by cardiac puncture and the animals 
were then sacrificed by cardiac excision. The blood samples were used for measuring plasma ALT and AST using assay kits. Liver tissue samples were obtained and subjected to biochemical analysis.

\subsection{Gentamicin Nephrotoxicity}

Gentamicin was administered intraperitoneally at a daily dose of $150 \mathrm{mg} / \mathrm{kg}$ from day 9 to day 14 during the course of BSS treatment (6 doses). Vehicle was given to the non-gentamicin-challenged animals. Twenty-four hours after the last gentamicin injection, blood samples were drawn from phenobarbital-anesthetized animals by cardiac puncture. The blood samples were used for measuring BUN and plasma creatinine levels, using assay kits. Animals were then sacrificed by cardiac excision. Both kidneys were harvested and subjected to biochemical analysis.

\subsection{Tissue ATP Level}

Tissue homogenates $(10 \%, \mathrm{w} / \mathrm{v})$ were prepared by homogenizing the minced tissues with a Teflon-glass homogenizer at $4000 \mathrm{rpm}$ for 10 complete strokes. An aliquot $(20 \mu \mathrm{L})$ of $30 \%(\mathrm{v} / \mathrm{v})$ perchloric acid (PCA) was added to $100 \mu \mathrm{L}$ of tissue homogenates. After neutralization by potassium bicarbonate, the ATP content was measured by bioluminescence assay. The data were normalized with respective protein content and expressed as nmol ATP per mg tissue protein.

\subsection{Mitochondrial Fraction Preparation}

Mitochondrial fractions were prepared by differential centrifugation in corresponding isotonic buffers (kidney: $320 \mathrm{mM}$ sucrose, $1 \mathrm{mM}$ ethylenediaminetetraacetic acid (EDTA), $50 \mathrm{mM}$ Tris/HCl, pH 7.4; liver: $250 \mathrm{mM}$ sucrose, $0.1 \mathrm{mM}$ EDTA, $5 \mathrm{mM}$ Tris/HCl, pH7.4), as previously described [43].

\subsection{Mitochondrial ATP-GC ex Vivo}

Mitochondrial fraction (adjusted to $1 \mathrm{mg}$ protein $/ \mathrm{mL}$ ) was mixed with substrate solution (3 $\mathrm{mM}$ pyruvate and $3 \mathrm{mM}$ malate) and ADP $(30 \mathrm{mM})$ solution to allow mitochondrial ATP generation. The ATP content was then measured as described [44].

\subsection{Mitochondrial Glutathione Redox Status}

Mitochondrial GSH and GSSG levels were measured enzymatically using 5,5'-dithiobis-(2nitrobenzonic acid) (DTNB) and GR as described [45]. The glutathione redox status was assessed by the ratio of GSH/GSSG.

\subsection{Mitochondrial GR and ICDH Activities}

Mitochondrial GR activity was measured as described by Chiu et al. (2003) [46]. For the measurement of mitochondrial ICDH activity, a sample mixture, which was prepared by mixing mitochondrial fraction $(100 \mu \mathrm{L}), 0.3 \%$ Triton-X $(100 \mu \mathrm{L})$ and phosphate-buffered saline $(100 \mu \mathrm{L})$, was sonicated at $4{ }^{\circ} \mathrm{C}$ for $10 \mathrm{~min}$. An aliquot $(120 \mu \mathrm{L})$ of reaction mixture $(5 \mathrm{mM}$ isocitrate, $0.435 \mathrm{mM}$ NADP and 1 drop of $\left.0.1 \mathrm{M} \mathrm{MnCl}_{2}\right)$ was added to the sample mixture $(60 \mu \mathrm{L})$. Absorbance changes at 
$340 \mathrm{~nm}$ were monitored at $37^{\circ} \mathrm{C}$ for $5 \mathrm{~min}$. ICDH activity was estimated by noting the absorption coefficient of NADPH.

\subsection{Statistical Analysis}

All data were expressed as mean \pm standard error of the mean (SEM), unless otherwise specified. Data were analyzed by one-way analysis of variance (one-way ANOVA) and inter-group difference was detected by Turkey range test when $p<0.05$.

\section{Conclusions}

In conclusion, BSS pretreatment protected against $\mathrm{CCl}_{4}$ hepatotoxicity but not gentamicin nephrotoxicity in rats. The hepatoprotection afforded by BSS against oxidant injury is likely mediated by the up-regulation of mitochondrial glutathione redox cycling via the induction of mitochondrial ROS production in rat livers. Therefore, long-term treatment with BSS may produce a mitohormetic action to prevent oxidative stress-induced oxidant injury in the liver.

\section{Supplementary Materials}

Supplementary materials can be accessed at: http:/www.mdpi.com/1420-3049/19/11/17649/s1.

\section{Author Contributions}

K.-M.K. supervised the study; H.-S.W. conceived and designed the experiments; H.-S.W., J.-H.C., P.-K.L., H.-Y.L. and W.-M.C. performed the experiments; K.-M.K. and H.-S.W. analyzed the data; H.-S.W. contributed reagents and materials; H.-S.W. wrote the paper. All authors have read and approved the manuscript.

\section{Abbreviations}

Alanine aminotransferase (ALT); aspartate aminotransferase (AST); ATP generation capacity (ATP-GC); $\beta$-sitosterol (BSS); blood urea nitrogen (BUN); carbon tetrachloride $\left(\mathrm{CCl}_{4}\right)$; 5,5'-dithiobis(2-nitrobenzoic acid) (DTNB); ethylenediaminetetraacetic acid (EDTA); glutathione reductase (GR); Cistanches Herba fraction (HCF1); isocitrate dehydrogenase (ICDH); oxidized glutathione (GSSG); perchloric acid (PCA); reactive oxygen species (ROS); reduced glutathione (GSH).

\section{Conflicts of Interest}

The authors declare no conflict of interest

\section{References}

1. Cesaratto, L.; Vascotto, C.; Calligaris, S.; Tell, G.L. The importance of redox state in liver damage. Ann. Hepatol. 2004, 3, 86-92.

2. Tanikawa, K.; Torimura, T. Studies on oxidative stress in liver diseases: Important future trends in liver research. Med. Mol. Morphol. 2006, 39, 22-27. 
3. Li, G.; Chen, Y.; Hu, H.; Liu, L.; Hu, X.; Wang, J.; Shi, W.; Yin, D. Association between age-related decline of kidney function and plasma malondialdehyde. Rejuvenation Res. 2012, 15, 257-264.

4. Small, D.M.; Coombes, J.S.; Bennett, N.; Johnson, D.W.; Gobe, G.C. Oxidative stress, anti-oxidant therapies and chronic kidney disease. Nephrology 2012, 17, 311-321.

5. Molina, M.F.; Sanchez-Reus, I.; Iqlesias, I.; Benedi, J. Quercetin, a flavonoid antioxidant, prevents and protects against ethanol-induced oxidative stress in mouse liver. Biol. Pharm. Bull. 2003, 26, 1398-1402.

6. Tirkey, N.; Pilkhwal, S.; Kuhad, A.; Chopra, K. Hesperidin, a citrus bioflavonoid, decreases the oxidative stress produced by carbon tetrachloride in rat liver and kidney. BMC Pharmacol. 2005, $5,2$.

7. Bejma, J.; Ramires, P.; Ji, L.L. Free radical generation and oxidative stress with ageing and exercise: Differential effects in the myocardium and liver. Acta Physiol. Scand. 2000, 169, 242-351.

8. Navarro, A.; Boveris, A. Rat brain and liver mitochondria develop oxidative stress and lose enzymatic activities on aging. Am. J. Physiol. Regul. Integr. Comp. Physiol. 2004, 287, R1244-R1249.

9. Hensley, K.; Robinson, K.A.; Gabbita, S.P.; Salsman, S.; Floyd, R.A. Reactive oxygen species, cell signaling, and cell injury. Free Radic. Biol. Med. 2000, 28, 1456-1462.

10. Finkel, T.; Holbrook, N.J. Oxidants, oxidative stress and the biology of ageing. Nature 2000, 408, 239-247.

11. Hou, F.; Zhang, R.; Zhang, M.; Su, D.; Wei, Z.; Deng, Y.; Zhang, Y.; Chi, J.; Tang, X. Hepatoprotective and antioxidant activity of anthocyanins in black rice bran on carbon tetrachloride-induced liver injury in mice. J. Funct. Foods 2013, 5, 1705-1713.

12. Khan, W.; Arivarasu, N.A.; Priyamvada, S.; Khan, S.A.; Khan, S.; Yusufi, A.N.K. Protective effect of $\omega-3$ polyunsatuated fatty acids (PUFA) on sodium nitrite induced nephrotoxicity and oxidative damage in rat kidney. J. Funct. Foods 2013, 5, 956-967.

13. Pinto, C.; Rodriguez-Galdon, B.; Cestero, J.J.; Macias, P. Hepatoprotective effects of lycopene against carbon tetrachloride-induced acute liver injury in rats. J. Funct. Foods 2013, 5, 1601-1610.

14. Chen, J.; Wong, H.S.; Leung, H.Y.; Leong, P.K.; Chan, W.M.; Chen, N.; Ko, K.M. An ursolic acid-enriched extract of Cynomorium songaricum protects against carbon tetrachloride hepatotoxicity and gentamicin nephrotoxicity in rats possibly through a mitochondrial pathway: A comparison with ursolic acid. J. Funct. Foods 2014, 7, 330-341.

15. Wong, H.S.; Ko, K.M. Herba Cistanches stimulates cellular glutathione redox cycling by reactive oxygen species generated from mitochondrial respiration in $\mathrm{H} 9 \mathrm{c} 2$ cardiomyocytes. Pharm. Biol. 2013, 51, 63-73.

16. Wong, H.S.; Chen, N.; Leong, P.K.; Ko, K.M. $\beta$-Sitosterol enhances cellular glutathione redox cycling by reactive oxygen species generated from mitochondrial respiration: Protection against oxidant injury in H9c2 cells and rat hearts. Phytother. Res. 2013, 28, 999-1006.

17. Forbes-Hernández, T.Y.; Giampieri, F.; Gasparrini, M.; Mazzoni, L.; Quiles, J.L.; Alvarez-Suarez, J.M.; Battino, M. The effects of bioactive compounds from plant foods on mitochondrial function: A focus on apoptotic mechanisms. Food Chem. Toxicol. 2014, 68, 154-182.

18. Giurgea-Marion, L.; Toubeau, G.; Lauent, G.; Heuson-Stiennon, J.A.; Tulkens, P.M. Impairment of lysosome-pinocytic vesicle fusion in rat kidney proximal tubules after treatment with gentamicin at low dose. Toxicol. Appl. Pharmacol. 1986, 86, 271-285. 
19. Beauchamp, D.; Gourde, P.; Bergeron, M.G. Subcellular distribution of gentamicin in proximal tubular cells, determined by immunogold labeling. Antimicrob. Agents Chemother. 1991, 35, 2173-2179.

20. Castillo, T.; Koop, D.R.; Kamimura, S.; Triadafilopoulos, G.; Tsukamoto, H. Role of cytochrome $\mathrm{P}-450$ 2E1 in ethanol-, carbon tetrachloride-and iron-dependent microsomal lipid peroxidation. Hepatology 1999, 16, 992-996.

21. Cabre, M.; Camps, J.; Patemain, J.L.; Ferre, N.; Joven, J. Time-course of changes in hepatic lipid peroxidation and glutathione metabolism in rats with carbon tetrachloride-induced cirrhosis. Clin. Exp. Pharmacol. Physiol. 2000, 27, 694-699.

22. Simile, M.M.; Banni, S.; Angioni, E.; Carra, G.; de Miglio, M.R.; Muroni, M.R.; Calvisi, D.F.; Carru, A.; Oascale, R.M.; Feo, F. 5'-Methylthiodenosine administration prevents lipid peroxidation and fibrogenesis induced in rat liver by carbon-tetrachloride intoxication. J. Hepatol. 2001, 24, 386-394.

23. Quiros, Y.; Vicente-Vicente, L.; Morales, A.I.; Lopez-Novoa, J.M.; Lopez-Hernandez, F.J. An integrative overview on the mechanisms underlying the renal tubular cytotoxicity of gentamicin. Toxicol. Sci. 2011, 119, 245-256.

24. Rebrin, I.; Sohal, R.S. Pro-oxidant shift in glutathione redox state during aging. Adv. Drug Deliv. Rev. 2008, 60, 1545-1552.

25. Babson, J.R.; Abell, N.S.; Reed, D.J. Protective role of the glutathione redox cycle against adriamycin-mediated toxicity in isolated hepatocytes. Biol. Pharm. 1981, 30, 2299-2304.

26. Harlan, J.M.; Levine, J.D.; Callahan, K.S.; Schwartz, B.R.; Harker, L.A. Glutathione redox cycle protects cultured endothelial cells against lysis by extracellularly generated hydrogen peroxide. J. Clin. Investig. 1984, 73, 106-713.

27. Rose, S.; Melnyk, K.; Pavliv, O.; Bai, S.; Nick, T.G.; Frye, R.E.; James, S.J. Evidence of oxidative damage and inflammation associated with low glutathione redox status in the autism brain. Transl. Psychiatry 2012, 2, e134.

28. Zhang, C.; Rodriguez, C.; Spaulding, J.; Aw, T.Y.; Feng, J. Age-dependent and tissue-related glutathione redox status in a mouse model of Alzheimer's Disease. J. Alzheimer's Dis. 2012, 28, 655-666.

29. Lluis, J.M.; Morales, A.; Blasco, C.; Colell, A.; Mari, M.; Garcia-Ruiz, C.; Fernandez-Checa, J.C. Critical role of mitochondrial glutathione in the survival of hepatocytes during hypoxia. J. Biol. Chem. 2005, 280, 3224-3232.

30. Schafer, F.Q.; Buettner, G.R. Redox environment of the cell as viewed through the redox state of the glutathione disulfide/glutathione couple. Free Radic. Biol. Med. 2001, 30, 1191-1212.

31. Fleury, C.; Mignotte, B.; Vayssiere, J.L. Mitochondrial reactive oxygen species in cell death signaling. Biochimie 2002, 84, 131-141.

32. Jo, S.H.; Son, M.K.; Koh, H.J.; Lee, S.M.; Song, I.H.; Kim, Y.O.; Lee, Y.S.; Jeong, K.S.; Kim, W.B.; Park, J.W.; et al. Control of mitochondrial redox balance and cellular defense against oxidative damage by mitochondrial $\mathrm{NADP}^{+}$-dependent isocitrate dehydrogenase. J. Biol. Chem. 2001, 276, 16168-16176.

33. Klein, J.A.; Ackerman, S.L. Oxidative stress, cell cycle, and neurodegeneration. J. Clin. Investig. 2003, 111, 785-793. 
34. Silva, A.H.; Fiqueiredo, L.M.; Dias, P.A.; Prado Neto, A.X.; Vasconcelos, P.R.; Guimaraes, S.B. Electroacupuncture attenuates liver and kidney oxidative stress in anesthetized rats. Acta Cir. Bras. 2011, 26, 60-65.

35. Zager, R.A. Adenine nucleotide changes in kidney, liver, and small intestine during different forms of ischemic injury. Circ. Res. 1991, 68, 185-196.

36. Devalaraja-Narashimha, K.; Singaravelu, K.; Padanilam, B.J. Poly (ADP-ribose) polymerase-mediated cell injury in acute renal failure. Pharmacol. Res. 2005, 52, 44-59.

37. Park, C.; Moon, D.O.; Rhu, .H.; Choi, B.T.; Lee, W.H.; Kim, G.Y.; Choi, Y.H. Beta-sitosterol induces anti-proliferation and apoptosis in human leukemic U937 cells through activation of caspase-3 and induction of Bax/Bcl-2 ratio. Biol. Pharm. Bull. 2007, 30, 1317-1323.

38. Schmucker, D.L. Liver function and phase I drug metabolism in the elderly. Drugs Aging 2001, $18,837-851$.

39. Frith, J.; Jones, D.; Newton, J.L. Chronic liver disease in an ageing population. Age Aging 2009, $38,11-18$.

40. Sheedfar, F.; Di Biase, S.; Koonen, D.; Vinciguerra, M. Liver diseases and aging: Friends or foes? Aging Cell 2013, 12, 950-954.

41. Sanders, D.J.; Minter, H.J.; Howes, D.; Hepburn, P.A. The safety evaluation of phytosterol esters. Part 6. The comparative absorption and tissue distribution of phytosterols in the rat. Food Chem. Toxicol. 2000, 38, 485-491.

42. Pharmacological Activities of Cistanches Herba on Kidney and Liver in Rats; Protocol \#2013051 (3 Years); The Government of Hong Kong Special Administrative Region, Department of Health: Hong Kong, 2013.

43. Leong, P.K.; Chen, N.; Chiu, P.Y.; Leung, H.Y.; Chung, W.M.; Tang, Q.T.; Ko, K.M. Long-term treatment with shengmai san-derived herbal supplement (Wei Kang Su) enhances antioxidant response in various tissues of rats with protection against carbon tetrachloride hepatotoxicity. J. Med. Food 2010, 13, 427-438.

44. Leung, H.Y.; Ko, K.M. Herba Cistanche extract enhances mitochondrial ATP generation in rat hearts and H9c2 cells. Pharm. Biol. 2008, 46, 418-424.

45. Leung, H.Y.; Chiu, P.Y.; Poon, M.K.T.; Ko, K.M. A Yang-invigorating Chinese herbal formula enhances mitochondrial functional ability and antioxidant capacity in various tissues of male and female rats. Rejuvenation Res. 2005, 8, 238-247.

46. Chiu, P.Y.; Tang, M.H.; Mak, D.H.; Poon, M.K.; Ko, K.M. Hepatoprotective mechanism of schisandrin B: Role of mitochondrial glutathione antioxidant status and heat shock proteins. Free Radic. Biol. Med. 2003, 35, 368-380.

Sample Availability: $\beta$-sitosterol (BSS) and BSS-containing HCF1 fraction are available from the authors.

(C) 2014 by the authors; licensee MDPI, Basel, Switzerland. This article is an open access article distributed under the terms and conditions of the Creative Commons Attribution license (http://creativecommons.org/licenses/by/4.0/). 